\title{
COVID-19 en el enfermo renal. Revisión breve
}

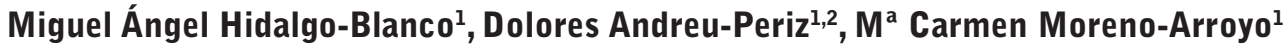 \\ ${ }^{1}$ Departamento de Enfermería Fundamental y Médico Quirúrgica. Facultad de Medicina y Ciencias de la Salud. \\ Universitat de Barcelona. España \\ ${ }^{2}$ Profesora Honorífica de la Universitat de Barcelona. España
}

Como citar este artículo: Hidalgo-Blanco MA, Andreu-Periz D, Moreno-Arroyo MC. COVID-19 en el enfermo renal. Revisión breve. Enferm Nefrol. 2020 Abr-Jun;23(2):122-31

\section{Resumen}

En diciembre de 2019 se identificó en China una nueva subespecie de coronavirus al que denominaron SARSCoV-2, responsable de la enfermedad posterior a la que la OMS Ilamó COVID-19. La enfermedad se ha propagado rápidamente provocando una pandemia mundial.

Todavía se desconoce mucho del SARS-CoV-2, pero las primeras investigaciones respaldan la hipótesis de que la gravedad de la COVID-19 viene condicionada por la respuesta hiperinflamatoria que se produce en nuestro organismo al contacto con el SARS-CoV-2. La gravedad del cuadro se relaciona con la insuficiencia respiratoria que provoca, no obstante, existen estudios que no limitan la afectación pulmonar. Investigaciones apuntan a que el mecanismo de acceso del SARS-CoV-2 al organismo está muy relacionado con la enzima ACE2. Enzima que entre otros tejidos, se puede encontrar en el epitelio de las células tubulares renales. Esta es la causa por la que existen datos de pacientes con COVID-19 que tienen una gran afectación en la función renal y pueden cursar con IRA (factor de mal pronóstico).

Por este motivo, unido a que las comorbilidades asociadas con una mayor mortalidad durante la infección COVID-19 son comunes en los pacientes con enfermedad renal crónica, creemos necesario conocer los resultados que aportan los diferentes estudios realizados sobre esa materia.

\section{Correspondencia:}

Miguel Ángel Hidalgo Blanco

E-mail: miguelhidalgo@ub.edu
PALABRAS CLAVE: COVID-19; SARS-CoV-2; enfermedades renales; diálisis; trasplante de riñón; revisión.

\section{COVID-19 in renal patient. Brief Review}

\section{Abstract}

In December 2019, a new subspecies of coronavirus was identified in China, which was named SARSCoV-2, responsible for the disease that WHO called COVID-19. The disease has spread rapidly causing a global pandemic.

Much is still unknown about SARS-CoV-2, but the first findings support the hypothesis that the severity of COVID-19 is conditioned by the hyperinflammatory response, which occurs in our body after contacting SARS-CoV-2. The severity of the symptoms is conditioned by the respiratory failure caused, however there are studies that are not limited to lung involvement. Evidence suggests that the access mechanism of the SARS-CoV-2 virus is closely related to the ACE2 enzyme. An enzyme that, among other tissues, can be found in the epithelium of renal tubular cells. For this reason, there are data on patients with COVID-19 who are severely affected in kidney function and may have acute kidney failure (a poor prognostic factor).

For this reason, together with the fact that the comorbidities associated with a higher mortality during $\mathrm{CO}$ VID-19 infection are common in patients with chronic 
kidney disease, it is necessary to know the available evidence on this matter.

KEYWORDS: D-19; SARS-CoV-2; kidney disease; dialysis; kidney transplant; review.

\section{Introducción}

En diciembre de 2019 en Wuhan, China, se identificaron a una serie de pacientes con enfermedad respiratoria aguda de origen desconocido ${ }^{1,2}$. Los distintos estudios detectaron que la enfermedad estaba causada por un nuevo betacoronavirus al que denominaron coronavirus 2 del síndrome respiratorio agudo grave (SARSCoV-2). El 11 de febrero de 2020, la Organización Mundial de la Salud cambió oficialmente el nombre de la enfermedad causada por el SARS-CoV-2 a enfermedad por coronavirus 2019 (COVID-19) y tras la rápida propagación mundial, fue declarada la pandemia3 ${ }^{3}$.

La enfermedad se propagó rápidamente desde Wuhan a otras áreas del mundo. En particular, Europa siguió como foco de enfermedad después de China, con Italia y España liderando el número total de casos. En España, el primer caso fue diagnosticado el 31 de enero de 2020 y, hasta la fecha, se han diagnosticado más de 227.000 casos.

Los coronavirus ( $\mathrm{CoV}$ ) son virus de ARN de cadena sencilla muy prevalentes en la naturaleza. Tienen muchos potenciales huéspedes naturales, intermedios y finales, fenómeno que plantea grandes desafíos para su tratamiento y la prevención de contagio ${ }^{4}$. Se conocen 7 subtipos de coronavirus que son infecciosos para los humanos y de estos, los betacoronavirus (beta-CoV) son los que están asociados con una enfermedad potencialmente grave. Dentro de este subtipo se encuentran el MERS-CoV y el SARS-CoV, virus que fueron responsables de brotes con morbilidad y mortalidad considerables en el pasado ${ }^{5}$. No obstante, lo que se ha podido descubrir en últimos estudios, es que la caracterización genómica del SARS-CoV-2 revela una distancia filogenética significativa de estos coronavirus previamente identificados, ya que comparte solo el $79 \%$ y el $50 \%$ de identidad con el SARS-CoV y el MERS-CoV, respectivamente ${ }^{2,6}$.

En comparación con el SARS-CoV y el MERS-CoV, el SARS-CoV-2 tiene una alta transmisibilidad e infectivi- dad, pero baja tasa de mortalidad ${ }^{7}$, no obstante, existen sospechas de que su tasa de mortalidad real puede no haberse estimado correctamente ${ }^{8}$.

Según los últimos estudios, la infección por SARS-CoV-2 comprende dos fases diferenciadas. Una primera fase que está asociada con la replicación viral y su efecto citopático, y una segunda fase que comenzaría después de 7-10 días desde el inicio de los síntomas y que se asocia con el riesgo de muerte9. Esta etapa se caracteriza por un compromiso pulmonar progresivo con necesidades crecientes de suplemento de oxígeno y soporte ventilatorio, que parece ser secundario a un síndrome hiperinflamatorio y derivado de la liberación de citoquinas ${ }^{10}$.

El manejo terapéutico de la enfermedad aún está en análisis y su enfoque todavía carece de evidencia significativa. Así como en la fase de replicación viral se pueden utilizar medicamentos antivirales como por ejemplo cloroquina-hidroxicloroquina, lopinavir/ritonavir, darunavir/ ritonavir y darunavir/cobicistat, la indicación para esta terapia antirretroviral es incierta y hasta la fecha no hay medicamentos aprobados para el tratamiento de la infección por SARS-CoV-2 ${ }^{11}$.

En la segunda fase de la enfermedad se utiliza un tratamiento de apoyo a la insuficiencia respiratoria que se produce por el Síndrome de Dificultad Respiratoria Aguda (SDRA), que es la causa principal de mortalidad ${ }^{12}$.

Recientes investigaciones relacionan el perfil de gravedad asociado a la enfermedad de COVID-19 con el síndrome derivado de la linfohistiocitosis hemofagocítica secundaria $(\mathrm{SHLH})^{13}$. La SHLH es un síndrome hiperinflamatorio poco conocido que se caracteriza por una hipercitoquinemia fulminante y mortal con insuficiencia multiorgánica. En la población adulta, se desencadena con mayor frecuencia por infecciones virales ${ }^{14} \mathrm{y}$ ocurre entre el $3,7 \%-4,3 \%$ de los casos de sepsis ${ }^{15}$. Entre sus manifestaciones se incluyen la fiebre mantenida, la citopenia y la hiperferritinemia. La afectación pulmonar (incluido el SDRA) ocurre en aproximadamente el 50\% de los pacientes que lo sufren ${ }^{16}$. En los casos de COVID-19, estas manifestaciones y otros hallazgos clínicos relacionados con el aumento de interleucinas, factor estimulante de colonias de granulocitos y proteínas inflamatorias, son predictores de gravedad ${ }^{9}$, por lo que las últimas investigaciones sugieren que la mortalidad podría deberse a esta hiperinflamación desencadenada por el virus ${ }^{12}$.

De la misma forma que durante las pandemias anteriores provocadas por el SARS-CoV y el MERS-CoV, los 
corticosteroides no se recomiendan de forma rutinaria y pueden exacerbar la lesión pulmonar asociada a COVID-1917. Sin embargo, en la hiperinflamación, es probable que los medicamentos inmunosupresores e inmunomoduladores podrían ser beneficiosos ${ }^{10}$. Por ello, algunos investigadores recomiendan someter a todos los pacientes con COVID-19 grave a pruebas serológicas de detección de hiperinflamación (aumento de ferritina, plaquetopenia o aumento de la velocidad de sedimentación globular) y a la escala de síndrome hemagofagocítico reactivo (HScore) ${ }^{18}$ para identificar a aquellos pacientes que se podrían beneficiar de terapia inmunosupresora y mejorar la mortalidad ${ }^{13}$.

\section{El riñón como órgano diana en la COVID-19}

A pesar de las diferencias antes descritas entre el SARSCoV-2 y otros coronavirus previamente identificados, varios estudios han informado que el SARS-CoV-2 al igual que el SARS-CoV explota la misma enzima convertidora de angiotensina unida a la membrana 2 (ACE2) para obtener acceso a sus células diana ${ }^{19-21}$, aunque a comparación el SARS-CoV-2 tiene una mayor afinidad de unión ${ }^{22,23}$. La ACE2 es una enzima que contrarresta el sistema de angiotensina-aldosterona (RAAS).

Un estudio reciente muestra que el ancestro común de estos dos virus es similar al coronavirus de murciélago HKU9- ${ }^{24}$. Estos tienen una estructura tridimensional de proteína espiga, que está estrechamente unida a la ACE2. Por lo tanto, las células con expresión de ACE2 pueden actuar como células diana y ser susceptibles a la infección por COVID-19, como las células alveolares tipo II (AT2) en el pulmón ${ }^{25}$. En este sentido también se ha demostrado que la proteína ACE2 tiene expresión en muchos otros tipos de células, como las células epiteliales intestinales, las células epiteliales tubulares renales, el corazón, las células del músculo liso de las arterias y las células gastrointestinales 26,27 .

En base a esta relación y dado el uso generalizado de los fármacos inhibidores de la enzima convertidora de angiotensina (IECA) para el control de la hipertensión o enfermedades cardiacas y renales, es necesario realizar más investigaciones para determinar si el agregar o suspender estos medicamentos puede alterar el curso de la infección aguda de COVID-19²8.

Pese a que existen estudios que afirman que es poco común que la infección por SARS-CoV-2 acabe en azotemia obvia e insuficiencia renal2 ${ }^{29,30}$, podemos afirmar, que el riñón humano puede ser un objetivo específico para la infección por SARS-CoV-2 ocasionando una insuficiencia renal aguda (IRA). El virus se puede unir a las células epiteliales renales, lesionándolas, alterando la homeostasis electrolítica y comprometiendo la regulación de la presión arterial. Además, existen evidencias de que la entrada viral en las células epiteliales renales puede posibilitar que el riñón también se convierta en un depósito viral, ocasionando que la orina sea un agente infeccioso ${ }^{31}$.

No obstante, la incidencia de IRA inducida por COVID-19 publicada hasta la fecha es muy variable. Estudios iniciales en China mostraban incidencias del $27 \%^{32}$ mientras que otros la disminuyen al 3,2\% ${ }^{33}$. Estas diferencias tan dispares se deben, en gran medida, a la gran variedad de estudios con muestras reducidas o con falta de homogeneidad entre las diferentes poblaciones.

Independientemente de estos datos, uno de los estudios destacados en el que más claramente se muestra una asociación entre la afectación renal y el mal resultado en pacientes con COVID-19, es el realizado en Wuhan por Cheng $Y$ et al. En una muestra de 701 pacientes ingresados con COVID-19, se detectaron elevaciones de creatinina sérica y BUN en el $14,4 \%$ y el $13,1 \%$ respectivamente. El $13,1 \%$ de los pacientes tenían una tasa de filtración glomerular estimada (TFG) $<60 \mathrm{ml} /$ $\mathrm{min} / 1,73 \mathrm{~m}^{2}$, el $43,9 \%$ de los pacientes tenían proteinuria y el $26,7 \%$ hematuria. En comparación con los pacientes con creatinina sérica normal, los que ingresaron con valores elevados, estaban más gravemente enfermos y tenían más probabilidades de ser ingresados en la unidad de cuidados intensivos y de necesitar ventilación mecánica. Además, demostraron mayor recuento leucocitario y un menor recuento de linfocitos y plaquetas. También, las anomalías en la vía de coagulación, incluido el tiempo prolongado de tromboplastina parcial activada y un dímero $D$ más elevado, fueron más comunes en pacientes con niveles elevados de creatinina sérica basal ${ }^{3}$.

Pese a la variabilidad de los resultados obtenidos en estos estudios, hay que destacar que la mayoría coinciden en el hecho de que la IRA grave en pacientes con COVID-19 es un predictor clínico de mal pronóstico, independientemente de la gravedad inicial de COVID-19 y de la condición física general. A pesar de este hallazgo significativo, se necesitarían más estudios para comprender los factores que se asocian a peores resultados entre los pacientes con COVID-19 con IRA, así como otros que nos permitan avanzar en la utilización de diferentes biomarcadores sensibles a la detección de la IRA (más allá de 
evaluar los aumentos en la creatinina sérica), para poder detectar casos de lesión renal subclínica asociada a las infecciones por COVID-1931,34.

\section{Enfermo renal y COVID-19}

Las comorbilidades asociadas con una mayor mortalidad durante la COVID-19 son comunes en los pacientes con enfermedad renal crónica (ERC) y en pacientes con trasplante de riñón o sometidos a terapia de reemplazo renal ${ }^{10}$. Sin embargo, algunas pequeñas series e informes de casos sugieren que la presentación clínica de estos pacientes renales podría ser leve, barajando la hipótesis de que sea a consecuencia del estado proinflamatorio que acusan. El enfermo renal crónico tiene una respuesta inmune deteriorada y por tanto existe una menor capacidad de desarrollar una tormenta de citoquinas ${ }^{35-39}$. No obstante, sí que se sabe que estos pacientes tienen un mayor riesgo de infección del tracto respiratorio superior $^{40}$ y de sufrir neumonías ${ }^{41}$.

\section{Enfermo renal en diálisis con COVID-19}

Dada las altas tasas de infección por SARS-CoV-2 en pacientes hospitalizados, la COVID-19 presenta desafíos para los pacientes en diálisis.

Los pocos estudios realizados hasta el momento se enfocan en observaciones de pequeños grupos y series, existiendo disparidad de opiniones. Una primera investigación realizada en un solo centro de hemodiálisis en el Hospital Renmin de la Universidad de Wuhan, reporta 37 casos de COVID-19 entre 230 pacientes de hemodiálisis. En la mayoría de estos pacientes, los síntomas de COVID-19 fueron leves, sin necesidad de ingreso en unidades de cuidados intensivos. En esta investigación se evidenció que estos pacientes tenían menos linfopenia y menores niveles séricos de citocinas inflamatorias que los pacientes sin diálisis afectados por la infección por COVID-19. Este estudio concluyó que los pacientes de hemodiálisis con COVID-19 son propensos a experimentar una enfermedad leve que no se desarrolla en una neumonía completa, probablemente debido a la función reducida del sistema inmune y a la disminución de las tormentas de citoquinas ${ }^{35}$.

Sin embargo, este hallazgo no concuerda con el encontrado en series italianas y españolas, en donde fallecieron alrededor de un $28 \%$ de los pacientes de hemodiálisis que requirieron ingreso hospitalario por COVID-1910,42. Además, al contrario de lo que se reporta de la investigación china, la fiebre y los síntomas respiratorios sí que fueron comunes en estos estudios. En estos casos se especula con la comorbilidad y los factores de riesgo de estos pacientes. Esta población, puede tener una prevalencia y una tasa de mortalidad muy altas para COVID-19, ya que combinan la edad avanzada, la desnutrición, la enfermedad cardiovascular, la diabetes, la enfermedad pulmonar y un sistema inmunitario menos eficiente con la necesidad de un tratamiento de diálisis en entornos superpoblados donde muchos pacientes, enfermeras, médicos y personal de apoyo están presentes al mismo tiempo ${ }^{43}$.

La elevación de la CRP, LDH, D-dímero y linfopenia fueron alteraciones bioquímicas que sí se encontraron en todos los estudios ${ }^{35,42}$.

Lo que se puede desprender de las diferentes series, es que aunque los síntomas de COVID-19 en pacientes en diálisis probablemente parezcan ser menos agresivos por su sistema inmunológico deteriorado, la mortalidad en estos pacientes puede ser más alta que en la población en general. Es obvio que en pacientes tan complejos y frágiles, una grave infección que se dirija a órganos como el pulmón y el corazón (ya comprometidos durante años de diálisis) conlleva a un riesgo muy alto de muerte, incluso si la propia infección no es la causa final${ }^{43}$.

Otro parámetro a tener en cuenta es que la infección por COVID-19 conduce a una inflamación sistémica y elevación de D-dímero, fragmentos proteicos asociados con la degradación de fibrina en coágulos sanguíneos. En este sentido, algunos autores recomiendan tener en cuenta esta alteración, ya que los dímeros $D$ pueden coagular los circuitos de diálisis, posiblemente más a menudo bajo condiciones de flujo sanguíneo bajo. Situación muy común durante los tratamientos de diálisis continua en pacientes hospitalizados. En este sentido será importante realizar planes para garantizar la seguridad de estos pacientes con COVID-19 y establecer pautas para dializarlos mejor ${ }^{44}$.

En los síndromes de disfunción multiorgánica, cuando el tratamiento farmacológico ya no es eficaz, las terapias de hemofiltración o hemoperfusión pueden ser posibilidades muy prometedoras. En estos casos, la oxigenación por membrana extracorpórea (ECMO) aplicada principalmente en modo veno-venoso, se ha destacado como la técnica más eficaz ${ }^{45-47}$. Las técnicas de depuración extracorpórea podrían usarse para eliminar factores inflamatorios, eliminar la tormenta de citoquinas, corregir el desequilibrio electrolítico y mantener el equilibrio ácido-base, para controlar la carga de capacidad del pacien- 
te de manera efectiva ${ }^{48}$. En esta lógica, los síntomas del paciente podrían mejorarse y podría aumentar la saturación de oxígeno en la sangre.

Además, en las instalaciones de hemodiálisis, la prevención y control de la enfermedad COVID-19 deberá ser prioritaria. Haciendo hincapié en el reconocimiento temprano de síntomas, el aislamiento de las personas con infección respiratoria, la colocación del paciente y el uso de equipos de protección personal. En este sentido, se han publicado varios artículos centrados en recomendaciones para contener la propagación de la infección en pacientes en diálisis y su personal sanitario con medidas generales y universales $44,49,50$; pero como se ha podido comprobar, se han publicado muy pocos informes sobre el grado real de difusión de la infección y su resultado en esta población específica.

\section{Manejo de la Enfermedad Glomerular ante la epidemia}

La COVID-19 también puede ser problemática para los pacientes con enfermedad glomerular que comenzaron terapia inmunosupresora antes de la pandemia. El Centro de Enfermedades Glomerulares de la Universidad de Columbia (Nueva York), remarca que en estos pacientes se debe tener en cuenta el riesgo-beneficio que supone la continuación de la terapia inmunosupresora. Se deben valorar factores como el impacto potencial de suspender o modificar el tratamiento, así como el acceso del paciente a dichas terapias. En los pacientes que estén en medio de un régimen de inducción intravenosa se pueden barajar la posibilidad del cambio a un régimen de inducción oral equivalente (si existe), para potenciar la adherencia en el hogar y evitar la exposición a entornos de atención médica ${ }^{51}$.

\section{COVID-19 en enfermos con Trasplante Renal}

Se percibe que los receptores de trasplante de riñón tienen un alto riesgo de infección, y el manejo de la terapia inmunosupresora durante esta emergencia global puede ser un desafío $0^{52}$. Sin embargo, las características clínicas, el enfoque terapéutico óptimo y los resultados de $\mathrm{CO}$ VID-19 entre los receptores de trasplantes de órganos sólidos siguen siendo en gran medida desconocidos ${ }^{53}$. La mayoría de investigaciones encontradas se refieren a estudios de casos y series pequeñas. En los casos concretos de trasplante renal, los datos con respecto a la letalidad asociada con el SARS-CoV-2 son escasos y con grupos muy pequeños ${ }^{37-39,54}$.

Uno de los informes de casos realizados en el Hospital 12 de Octubre (Madrid) sobre el impacto de la COVID-19 en los receptores de trasplantes de órganos sólidos, evidenció que existían tasas más altas de neumonía $(72,2 \%$ [13/18] versus $31,1 \%)$, SDRA $(38,9 \%$ [7/18] versus $5,6 \%)$ y admisión en la UCI ( $11,1 \%$ [2/18] versus 5,1\%) en comparación con la población general española ${ }^{55}$. No obstante, como en la mayoría de estudios realizados en este tipo de población, hay que tener cautela al interpretarlos debido a la heterogeneidad de ambos conjuntos de datos y a la pequeña muestra de estudio (18 pacientes). También, hay que destacar la alta tasa de letalidad encontrada en este estudio $(27,8 \%)$ en comparación con la de la población general, que coincide con la reportada por los informes de otros casos publicados en China, Italia y Nueva York ${ }^{37-39,56-61}$ y refutando la hipótesis de que la inmunosupresión a largo plazo pudiera ejercer un efecto protector ${ }^{62}$.

Los pacientes inmunodeprimidos presentan comúnmente signos y síntomas de infección atípicos 0 atenuados, que a menudo conducen a presentaciones tardías, o diagnósticos erróneos y peores resultados en general ${ }^{63}$. Los reportes de los diferentes casos de pacientes receptores de trasplante renal con COVID-19 ponen de manifiesto que estos pacientes parecen presentar menos fiebre y más síntomas gastrointestinales ${ }^{42}$, entre los que destaca la diarrea (síntoma cada vez más relacionado con el COVID-19)61. En este sentido, Fernández-Ruiz et al. en su estudio de casos en Madrid, cuestiona si la inmunosupresión derivada del trasplante puede modificar la presentación clínica de COVID-19 y, por tanto, ocasionar que estos pacientes trasplantados sean más propensos a presentar problemas gastrointestinales en comparación al resto de población 65 .

Lo que sí se ha podido observar es que los pacientes trasplantados con COVID-19 necesitan mucho más tiempo en volverse negativos para el virus. La inmunosupresión conlleva a una enfermedad que puede ser más grave y a una eliminación del virus que se prolongue más en el tiempo ${ }^{61}$.

Por todos estos motivos, se entiende que el manejo óptimo de la inmunosupresión en receptores de trasplante con COVID-19 sigue siendo incierto a pesar de la importancia y urgencia de esta cuestión. La inmunosupresión puede estar asociada con un control virológico deficiente, pero reducirla puede conducir a un rechazo agudo del trasplante.

Por tanto, debido a los datos limitados presentados en los casos, es difícil sacar conclusiones sobre el manejo óptimo de inmunosupresores en los receptores de trasplante renal. Una revisión de la literatura realizada por Johnson et al. define que la mayoría de los casos 
de pacientes con trasplante renal y con COVID-19, han realizado monoterapia con corticosteroides, sin embargo no se recomienda su uso rutinario y general para tratar pacientes infectados por SARS-CoV-2 ${ }^{64}$.

\section{Conclusiones}

Todavía nos encontramos en los momentos iniciales de la pandemia y nos queda mucho recorrido para conocer en profundidad todo lo que implica la infección por SARSCoV-2.

La mayoría de investigaciones realizadas han sido estudios de casos o de series muy pequeñas con características muestrales muy heterogéneas. Este motivo ocasiona disparidad de resultados o que no sean del todo concluyentes. Además, por los estudios reportados, la infección por SARS-CoV-2 tiene un comportamiento atípico, complicando su manejo y tratamiento, hecho que también dificulta la investigación y el consenso en la comunidad científica.

El origen de los casos graves de COVID-19 puede estar relacionado por una reacción hiperinflamatoria del organismo ante el SARS-CoV-2, por lo que las terapias inmunosupresoras o inmunomoduladoras podrían ser be- neficiosas. Como se ha visto, hay indicios de que la COVID-19 se comporta diferente en pacientes inmmunodeprimidos (como los pacientes trasplantados), que en el resto de población. Por tanto, parece claro que esta será una línea de investigación a desarrollar.

Además, parece claro que las células con expresión de ACE2 pueden actuar como células diana para el virus, y entre estas se encuentran las células epiteliales tubulares. Por este motivo, parece que el riñón podría ser un órgano objetivo para el virus, pudiendo llegar a provocar IRA en los casos más graves y con peor pronóstico.

Por todo esto, es de imperiosa importancia el continuar con las investigaciones sobre el SARS-CoV-2 y su relación con el paciente nefrológico, para poder llegar a conocer mejor la afectación renal que ocasiona y así desarrollar óptimos tratamientos preventivos y terapéuticos.

\begin{tabular}{|c|}
\hline Recibido: $04-05-20$ \\
Revisado: 06-05-20 \\
Modificado: $12-05-20$ \\
Aceptado: $14-05-20$
\end{tabular}

\section{Conceptos Clave}

La infección por SARS-CoV-2 comprende dos fases diferenciadas. Una primera fase que está asociada con la replicación viral y su efecto citopático, y una segunda fase que comenzaría después de 7-10 días desde el inicio de los síntomas y que se asocia con un estado de hiperinflamación.

EI SARS-CoV-2 explota la enzima convertidora de angiotensina unida a la membrana 2 (ACE2) para obtener acceso a sus células objetivo.

La proteína ACE2 tiene expresión en muchos tipos de células, como las células alveolares tipo II (AT2) en el pulmón y las células epiteliales tubulares renales.

La IRA grave en pacientes con COVID-19 es un predictor clínico de mal pronóstico independientemente de la gravedad inicial de COVID-19.

Aunque los síntomas de COVID-19 en pacientes en diálisis probablemente parezcan ser menos agresivos, la mortalidad en estos pacientes puede ser más alta que en la población en general.

La infección por COVID-19 conduce a una inflamación sistémica y los dímeros D pueden coagular los circuitos de diálisis, posiblemente más a menudo bajo condiciones de flujo sanguíneo bajo.

En los pacientes con enfermedad glomerular que comenzaron terapia inmunosupresora antes de la pandemia se debe tener en cuenta el riesgo-beneficio que supone la continuación de la terapia inmunosupresora.

Los estudios coinciden en que existe una alta tasa de letalidad en los pacientes trasplantados con COVID-19 en comparación con la de la población general.

Los reportes de los diferentes casos de pacientes receptores de trasplante renal con COVID-19 ponen de manifiesto que estos pacientes parecen presentar menos fiebre y más síntomas gastrointestinales (diarrea). Esto puede ser debido a la inmunosupresión que presentan. 


\section{Bibliografía}

1. Zhu N, Zhang D, Wang W, Li X, Yang B, Song J, et al. A Novel Coronavirus from Patients with Pneumonia in China, 2019. N Engl J Med 2020;382:727-33.

2. Lu R, Zhao X, Li J, Niu P, Yang B, Wu H, et al. Genomic characterisation and epidemiology of 2019 novel coronavirus: implications for virus origins and receptor binding. Lancet. 2020 Feb;395(10224):565-74.

3. Cheng $Y$, Luo R, Wang $K$, Zhang M, Wang Z, Dong $L$, et al. Kidney disease is associated with in-hospital death of patients with COVID-19. Kidney Int. 2020;97(5):829-38.

4. Li-sheng Wang, Yi-ru Wang, Da-wei Ye, Qing-quan Liu. A review of the 2019 Novel Coronavirus (COVID-19) based on current evidence. Int J Antimicrob Agents. 2020 Mar:105948. [En prensa].

5. Sun $P, L u X, X u C$, Sun W, Pan B. Understanding of COVID-19 based on current evidence. J Med Virol. 2020 Feb. [En prensa].

6. Wu A, Peng $Y$, Huang B, Ding $X$, Wang $X$, Niu $P$, et al. Genome composition and divergence of the novel coronavirus (2019-nCoV) originating in China. Cell Host \& Microbe. 2020 Feb;27(3):325-8.

7. Liu Y, Gayle AA, Wilder-Smith A, Rocklöv J. The reproductive number of COVID-19 is higher compared to SARS coronavirus. J Travel Med. 2020;27(2):taaa021.

8. Michaels MG, La Hoz RM, Danziger-Isakov L, Blumberg EA, Kumar D. Green M, et al. Coronavirus disease 2019: Implications of emerging infections for transplantation. Am J Transplant. 2020 Feb. [En prensa].

9. Huang C, Wang Y, Li X, Ren L, Zhao J, Hu Y, et al. Clinical features of patients infected with 2019 novel coronavirus in Wuhan, China. Lancet. 2020;395:497506.

10. Alberici $F$, Delbarba E, Manenti $C$, Econimo L, Valerio F, Pola A, et al. On behalf of the "Brescia Renal COVID Task Force, Management Of Patients On Dialysis And With Kidney Transplant During SARSCOV-2 (COVID-19) Pandemic In Brescia, Italy. Kidney Int Rep. 2020 Abr. [En prensa].
11. Wei LQ, Rong ZK, Gui L, Shan RD. CT diagnosis of renal vein thrombosis in nephrotic syndrome. $\mathrm{J}$ Comput Assist Tomogr. 1991;15(3):454-7.

12. Ruan $Q$, Yang $K$, Wang W, Jiang $L$, Song J. Clinical predictors of mortality due to COVID-19 based on an analysis of data of 150 patients from Wuhan, China. Intensive Care Med. 2020 May;46(5):846-8.

13. Mehta $P$, McAuley DF, Brown M, Sanchez E, Tattersall RS, Manson JJ (2020) COVID 19: consider cytokine storm syndromes and immunosuppression. Lancet. 2020 Mar;395(10229):1033-4.

14. Ramos-Casals $M$, Brito-Zeron $P$, Lopez-Guillermo $A$, Khamashta MA, Bosch X. Adult haemophagocytic syndrome. Lancet. 2014 Abr;383(9927):1503-16.

15. Karakike E, Giamarellos-Bourboulis EJ. Macrophage activation-like syndrome: a distinct entity leading to early death in sepsis. Front Immunol 2019;10:55.

16. Seguin A, Galicier L, Boutboul D, Lemiale V, Azoulay E. Pulmonary involvement in patients with hemophagocytic lymphohistiocytosis. Chest 2016;149:1294-301.

17. Russell CD, Millar JE, Baillie JK. Clinical evidence does not support corticosteroid treatment for 2019-nCoV lung injury. Lancet 2020;395(10223): 473-5.

18. Fardet $L$, Galicier L, Lambotte 0 , Marzac $C$, Aumont C, Chahwan D, et al. Development and validation of the HScore, a score for the diagnosis of reactive hemophagocytic syndrome. Arthritis Rheumatol 2014;66(9)2613-20.

19. Hoffmann M, Kleine-Weber $H$, Schroeder $S$, Krüger N, Herrler T, Erichsen S, et al. SARSCoV- 2 cell entry depends on ACE2 and TMPRSS2 and is blocked by a clinically proven protease inhibitor. Cell. 2020;181:271-80.

20. Yan R, Zhang $Y$, Li Y, Xia L, Guo $Y$, Zhou Q. Structural basis for the recognition of the SARS-CoV-2 by full-length human ACE2. Science. 2020 Mar; 367(6485):1444-8.

21. Liu Z, Xiao X, Wei X, Li J, Yang J, Tan H, et al. Composition and divergence of coronavirus spike proteins 
and host ACE2 receptors predict potential intermediate hosts of SARSCoV- 2. J Med Virol. 2020 Feb. [En prensa].

22. Wrapp D, Wang N, Corbett KS, Goldsmith JA, Hsieh $\mathrm{CL}$, Abiona 0, et al. Cryo-EM structure of the 2019$\mathrm{nCoV}$ spike in the prefusion conformation. Science. 2020 Feb;367(6483):1260-3.

23. Wan Y, Shang J, Graham R, Baric RS, Li F. Receptor Recognition by the Novel Coronavirus from Wuhan: an Analysis Based on Decade-Long Structural Studies of SARS Coronavirus. J Virol. 2020;94(7):e0012720.

24. Xu X, Chen P, Wang J, Feng J, Zhou H, Li X, et al. Evolution of the novel coronavirus from the ongoing Wuhan outbreak and modeling of its spike protein for risk of human transmission. Sci China Life Sci. 2020 Mar;63(3):457-60.

25. Zou X, Chen K, Zou J, Han P, Hao J, Han Z. Single-cell RNA-seq data analysis on the receptor ACE2 expression reveals the potential risk of different human organs vulnerable to 2019-nCoV infection. Front Med. 2020 Mar. [En prensa].

26. Ye M, Wysocki J, William J, Soler MJ, Cokic I, Batlle D. Glomerular localization and expression of angiotensin-converting enzyme 2 and angiotensin-converting enzyme: implications for albuminuria in diabetes. J Am Soc Nephrol. 2006 Nov;17(11):3067-75.

27. Wang L, Li X, Chen H, Yan S, Li D, Li Y, Gong Z. Coronavirus disease 19 infection does not result in acute kidney injury: an analysis of 116 hospitalized patients from Wuhan, China. Am J Nephrol. 2020;51(5):343-8.

28. Vaduganathan $M$, Vardeny 0 , Michel $T$, McMurray JJV, Pfeffer MA, Solomon SD. Renin-Angiotensin-Aldosterone System Inhibitors in Patients with Covid-19 N Engl J Med. 2020;382:1653-9.

29. Wang D, Hu B, Hu C, Zhu F, Liu X, Zhang J, et al. Clinical Characteristics of 138 Hospitalized Patients with 2019 Novel Coronavirus- Infected Pneumonia in Wuhan, China. JAMA. 2020;323(11):1061-9.

30. Guan WJ, Ni ZY, Hu Y, Liang WH, Ou CQ, He JX, et al. Clinical characteristics of 2019 novel coronavirus infection in China. N Engl J Med 2020; 382:1708-20.
31. Rabb H. Kidney diseases in the time of COVID-19: major challenges to patientcare. J Clin Invest. 2020 May. pii: 138871. [En prensa].

32. Diao B, Wang C, Wang R, Feng $Z$, Tan $Y$, Wang $H$, et al. Human Kidney is a Target for Novel Severe Acute Respiratory Syndrome Coronavirus 2 (SARS-CoV-2) Infection. medRxiv. 2020 Mar. [En prensa].

33. Cheng $Y$, Luo R, Wang $K$, Zhang M, Wang Z, Dong $L$, et al. Kidney impairment is associated with in-hospital death of COVID-19 patients. medRxiv. 2020 Feb. [En prensa].

34. Zhang WR, and Parikh CR. Biomarkers of Acute and Chronic Kidney Disease. Annu Rev Physiol. 2019;81:309-33.

35. Ma Y, Diao B, Lv X, Zhu J, Liang W, Wendou LL, et al. 2019 novel coronavirus disease in hemodialysis (HD) patients: Report from one HD center in Wuhan, China. medRxiv. 2020 Feb. [En prensa].

36. Wang $\mathrm{R}$, Liao $\mathrm{C}$, He $\mathrm{H}$, Hu C, Wei $\mathrm{Z}$, Hong $\mathrm{Z}$, et al. COVID-19 in Hemodialysis Patients: A Report of 5 Cases. Am J Kidney Dis. 2020 Mar. pii: S02726386(20)30612-0. [En prensa].

37. Guillen $E$, Pineiro GJ, Revuelta I, Rodriguez D, Bodro $M$, Moreno A, et al. Case report of COVID-19 in a kidney transplant recipient: Does immunosuppression alter the clinical presentation?. Am J Transplant. 2020 Mar. [En prensa].

38. Gandolfini I, Delsante M, Fiaccadori E, Zaza G, Manenti $L$, Antoni AD, et al. COVID-19 in Kidney Transplant Recipients. Am J Transplant. 2020;10.1111/ ajt.15891. [En prensa].

39. Zhu L, Xu X, Ma K, Yang K, Guan H, Chen S, et al. Successful recovery of COVID-19 pneumonia in a renal transplant recipient with long-term immunosuppression. Am J Transplant. 2020 Mar. [En prensa].

40. Cohen-Hagai K, Rozenberg I, Korzets Z, Zitman-Gal $T$, Einbinder $Y$, Benchetrit S. Upper Respiratory Tract Infection among Dialysis Patients. Isr Med Assoc J. 2016;18:557-60.

41. Sibbel S, Sato R, Hunt A, Tuernne W, Brurnelli SM. The clinical and economic burden of pneumonia in patients enrolled in Medicare receiving dialysis: 
a retrospective, observational cohort study. BMC Nephrol. 2016;17:199.

42. Trujillo H, Caravaca-Fontán $F$, Sevillano Á, Gutiérrez E, Caro J, Gutiérrez E, et al. SARS-CoV-2 Infection in Hospitalized Patients with Kidney Disease. Kidney Int Rep. May 2020. [En prensa].

43. Rombolà G, Brunini F. COVID-19 and dialysis: why we should be worried. J Nephrol. 2020 Abr;22:1-3.

44. Naicker S, Yang CW, Hwang SJ, Liu BC, Chen JH, Jha V.The Novel Coronavirus 2019 epidemic and kidneys. Kidney Int. 2020;97(5):824-28.

45. Brodie D, Curtis JR, Vincent JL, Bakker J, Brown CE, Creteur J, et al. Participants in the Round Table Conference. Treatment limitations in the era of ECM0. Lancet Respir Med. 2017 0ct;5(10):76970.

46. Noah MA, Peek GJ, Finney SJ, Griffiths MJ, Harrison $D A$, Grieve $R$, et al. Referral to an extracorporeal membrane oxygenation center and mortality among patients with severe 2009 influenza A(H1N1). JAMA. 2011 0ct; 306(15):1659-68.

47. DeLaney E, Smith MJ, Harvey BT, Pelletier KJ, Aquino MP, Stone JM, et al. Extracorporeal life support for pandemic influenza: the role of extracorporeal membrane oxygenation in pandemic management. J Extra Corpor Technol. 2010 Dic;42(4):268-80.

48. Lim CC, Tan CS, Kaushik M, Tan HK. Initiating acute dialysis at earlier Acute Kidney Injury Network stage in critically ill patients without traditional indications does not improve outcome: a prospective cohort study. Nephrology (Carlton). 2015;20:148-54.

49. Rombolà $G$, Heidempergher $M$, Pedrini $L$, Farina $M$, Aucella $F$, Messa $P$, et al. Practical indications for the prevention and management of SARS-CoV2 in ambulatory dialysis patients: lessons from the first phase of the epidemics in Lombardy. J Nephrol. 2020 Abr;33(2):193-6.

50. Ikizler TA. COVID-19 and dialysis units: what do we know now and what should we do?. Am J Kidney Dis. 2020 Mar. [En prensa].
51. Bomback AS, Canetta PA, Ahn W, Ahmad SB, Radhakrishnan J, Appel GB. How COVID-19 Has Changed the Management of Glomerular Diseases. CJASN. 2020 May. [En prensa].

52. Bussalino E, De Maria A, Russo R, Paoletti E. Immunosuppressive therapy maintenance in a kidney transplant recipient SARS-CoV-2 pneumonia: a case report. Am J Transplant. 2020 Abr. [En prensa].

53. Michaels MG, La Hoz RM, Danziger-Isakov L, Blumberg EA, Kumar D, Green $M$, et al. Coronavirus disease 2019: Implications of emerging infections for transplantation. Am J Transplant. 2020 Feb. [En prensa].

54. Alberici F, Delbarba E, Manenti C, Econimo L Valerio F, Pola A, et al. Management Of Patients On Dialysis And With Kidney Transplant During SARS-COV-2 (COVID-19) Pandemic In Brescia, Italy. Kidney Int Rep. 2020 Abr. [En prensa].

55. Fernández-Ruiz $M$, Andrés $A$, Loinaz $C$, Delgado JF, López-Medrano F, San Juan R, et al. COVID-19 in solid organ transplant recipients: a single-center case series from Spain. Am J Transplant. 2020 Abr. [En prensa].

56. Liu Bl, Wang Y, Zhao Y, Shi H, Zeng F, Chen Z. Successful treatment of severe COVID-19 pneumonia in a liver transplant recipient. Am J Transplant. 2020 Abr. [En prensa].

57. Seminari E, Colaneri M, Sambo M, Gallazzi I, Di Matteo A, Roda S, et al. SARS Cov2 infection in a renal transplanted patients. A case report. Am J Transplant. 2020 Abr. [En prensa].

58. Li F, Cai J, Dong N. First cases of COVID-19 in heart transplantation from China. J Heart Lung Transplant. 2020 Mar. [En prensa].

59. Chen S, Yin Q, Shi H, Du D, Chang S, Ni L, et al. A familial cluster, including a kidney transplant recipient, of Coronavirus Disease 2019 (COVID-19) in Wuhan, China. Am J Transplant. 2020 Abr. [En prensa].

60. Huang J, Lin $H$, Wu $Y$, Fang $Y$, Kumar R, Chen G, et al. COVID-19 in post-transplantation patients - report of two cases. Am J Transplant. 2020 Abr. [En prensa]. 
61. Pereira MR, Mohan S, Cohen DJ, Husain SA, Dube G, Ratner LE, et al., COVID-19 in Solid Organ Transplant Recipients: Initial Report from the US epicenter. Am J Transplant. 2020 Abr. [En prensa].

62. Antonio R, Silvia M. Immunosuppression drug-related and clinical manifestation of Coronavirus disease 2019: a therapeutical hypothesis. Am J Transplant. 2020 Abr. [En prensa].

63. Fishman, J.A. Infection in Organ Transplantation. Am J Transplant 2017;17(4): 856-79.

64. Johnson KM, Belfer JJ, Peterson GR, Boelkins MR, Dumkow LE. Managing COVID-19 in Renal Transplant Recipients: A Review of Recent Literature and Case Supporting Corticosteroid-sparing Immunosuppression. Pharmacotherapy. 2020;10.1002/ phar.2410. [En prensa].

Este artículo se distribuye bajo una Licencia Creative Commons Atribución-NoComercial 4.0 Internacional. https://creativecommons.org/licenses/by-nc/4.0/

\section{Open Access (c) (7) (8)}

